\title{
Early emergence of the FtsH proteases involved in photosystem II repair
}

\author{
S. SHAO, T. CARDONA, and P.J. NIXON ${ }^{+}$ \\ Department of Life Sciences, Sir Ernst Chain Building - Wolfson Laboratories, Imperial College London, South Kensington \\ Campus, London SW7 2AZ, UK
}

\section{Erratum to: Photosynthetica \\ DOI: 10.1007/s11099-018-0769-9}

\begin{abstract}
Page 1
Abstract

The verb „make“ was used instead of „form“ in the following sentence.

Instead of

A phylogenetic analysis of over $6000 \mathrm{FtsH}$ protease sequences revealed that there are three major groups of FtsH proteases originating from gene duplication events in the last common ancestor of bacteria, and that the FtsH proteases involved in PSII repair make a distinct clade branching out before the divergence of FtsH proteases found in all groups of anoxygenic phototrophic bacteria.

It should read

A phylogenetic analysis of over $6000 \mathrm{FtsH}$ protease sequences revealed that there are three major groups of FtsH proteases originating from gene duplication events in the last common ancestor of bacteria, and that the FtsH proteases involved in PSII repair form a distinct clade branching out before the divergence of FtsH proteases found in all groups of anoxygenic phototrophic bacteria.
\end{abstract}

The word „photosynthetic“ was omitted in the following sentence:

Instead of

We conclude that the phylogeny of FtsH proteases is consistent with an early origin of water oxidation chemistry.

It should read

We conclude that the phylogeny of FtsH proteases is consistent with an early origin of photosynthetic water oxidation chemistry.

\section{Introduction}

The words „reactions“ and „for“ were used mistakenly in the following paragraph:

Instead of

Oxygenic photosynthetic electron transport from water to $\mathrm{NADP}^{+}$requires the participation of two functionally distinct reactions centers (RCs) acting in series: photosystem II (PSII, a Type II RC containing quinone electron acceptors) and photosystem I (PSI, a Type I RC containing redox-active iron-sulphur clusters). Early ideas on for the emergence of oxygenic photosynthesis focused on the evolution of PSI and PSII from pre-existing RCs found in anoxygenic photosynthetic bacteria (Nitschke and Rutherford 1991) and the horizontal transfer of genes encoding chlorophyll biosynthetic enzymes and RC proteins (Hohmann-Marriott and Blankenship 2011, Fischer et al. 2016).

It should read

Oxygenic photosynthetic electron transport from water to $\mathrm{NADP}^{+}$requires the participation of two functionally distinct reaction centers (RCs) acting in series: photosystem II (PSII, a Type II RC containing quinone electron 
acceptors) and photosystem I (PSI, a Type I RC containing redox-active iron-sulphur clusters). Early ideas on the emergence of oxygenic photosynthesis focused on the evolution of PSI and PSII from pre-existing RCs found in anoxygenic photosynthetic bacteria (Nitschke and Rutherford 1991) and the horizontal transfer of genes encoding chlorophyll biosynthetic enzymes and RC proteins (Hohmann-Marriott and Blankenship 2011, Fischer et al. 2016).

\section{Page 12}

The word „other“ was missing in the following sentence:

Instead of

Possible evolutionary constraints include the need to maintain protein quality control in the multiple membrane compartments found in cyanobacteria, chloroplasts, and mitochondria and the fact that oxygenic photosynthesis is associated with the production of singlet oxygen and ROS (reactive oxygen species), leading to protein damage, particularly in the thylakoid membrane which houses the photosynthetic apparatus.

It should read

Possible evolutionary constraints include the need to maintain protein quality control in the multiple membrane compartments found in cyanobacteria, chloroplasts and mitochondria and the fact that oxygenic photosynthesis is associated with the production of singlet oxygen and other ROS (reactive oxygen species), leading to protein damage, particularly in the thylakoid membrane which houses the photosynthetic apparatus.

The word „of“" was mistakenly used instead of „to“ in the following sentence:

Instead of

Recognition of damaged D1 by cyanobacterial FtsH complexes is thought to be mediated by partial disassembly of damaged PSII (Krynická et al. 2015) and binding of the N-terminal tail of D1 (Komenda et al. 2007).

It should read

Recognition of damaged D1 by cyanobacterial FtsH complexes is thought to be mediated by partial disassembly of damaged PSII (Krynická et al. 2015) and binding to the N-terminal tail of D1 (Komenda et al. 2007).

The online version of the original article can be found under DOI: $10.1007 / \mathrm{s} 11099-018-0769-9$

${ }^{+}$Corresponding author; e-mail: p.nixon@imperial.ac.uk

The publisher and authors apologize for these errors and for inconveniences they may have caused. 